\title{
Author Correction: Reference component analysis of single-cell transcriptomes elucidates cellular heterogeneity in human colorectal tumors
}

Huipeng Li, Elise T Courtois, Debarka Sengupta, Yuliana Tan, Kok Hao Chen, Jolene Jie Lin Goh, Say Li Kong, Clarinda Chua, Lim Kiat Hon, Wah Siew Tan, Mark Wong, Igor Cima, Min-Han Tan, Lawrence J K Wee, Axel M Hillmer, lain Beehuat Tan, Paul Robson and Shyam Prabhakar

Correction to: Nature Genetics https://doi.org/10.1038/ng.3818, published online 20 March 2017.

In the version of the article published, the author list is not accurate. Igor Cima and Min-Han Tan should have been authors, appearing after Mark Wong in the author list, while Paul Jongjoon Choi should not have been listed as an author. Igor Cima and Min-Han Tan both have the affiliation Institute of Bioengineering and Nanotechnology, Singapore, Singapore, and their contributions should have been noted in the Author Contributions section as "I.C. preprocessed Primary Cell Atlas data with inputs from M.-H.T." The following description of the contribution of Paul Jongjoon Choi should not have appeared: "P.J.C. supported the smFISH experiments." In the 'RCA: global panel' section of the Online Methods, the following sentence should have appeared as the second sentence, "An expression atlas of human primary cells (the Primary Cell Atlas) was preprocessed similarly to in ref. 55," with new reference 55 (Cima, I. et al. Tumor-derived circulating endothelial cell clusters in colorectal cancer. Science Transl. Med. 8, 345ra89, 2016).

\section{Publisher Correction: Allele-defined genome of the autopolyploid sugarcane Saccharum spontaneum $\mathrm{L}$}

Jisen Zhang (D), Xingtan Zhang, Haibao Tang (D), Qing Zhang, Xiuting Hua, Xiaokai Ma, Fan Zhu, Tyler Jones, Xinguang Zhu, John Bowers, Ching Man Wai, Chunfang Zheng, Yan Shi, Shuai Chen, Xiuming Xu, Jingjing Yue, David R. Nelson, Lixian Huang, Zhen Li, Huimin Xu, Dong Zhou, Yongjun Wang, Weichang Hu, Jishan Lin, Youjin Deng, Neha Pandey, Melina Mancini, Dessireé Zerpa, Julie K. Nguyen, Liming Wang, Liang Yu, Yinghui Xin, Liangfa Ge, Jie Arro, Jennifer O. Han, Setu Chakrabarty, Marija Pushko, Wenping Zhang, Yanhong Ma, Panpan Ma, Mingju Lv, Faming Chen, Guangyong Zheng, Jingsheng Xu, Zhenhui Yang, Fang Deng, Xuequn Chen, Zhenyang Liao, Xunxiao Zhang, Zhicong Lin, Hai Lin, Hansong Yan, Zheng Kuang, Weimin Zhong, Pingping Liang, Guofeng Wang, Yuan Yuan, Jiaxian Shi, Jinxiang Hou, Jingxian Lin, Jingjing Jin, Peijian Cao Di , Qiaochu Shen, Qing Jiang, Ping Zhou, Yaying Ma, Xiaodan Zhang, Rongrong Xu, Juan Liu, Yongmei Zhou, Haifeng Jia, Qing Ma, Rui Qi, Zhiliang Zhang, Jingping Fang, Hongkun Fang, Jinjin Song, Mengjuan Wang, Guangrui Dong, Gang Wang, Zheng Chen, Teng Ma, Hong Liu, Singha R. Dhungana (1), Sarah E. Huss, Xiping Yang, Anupma Sharma, Jhon H. Trujillo, Maria C. Martinez (D), Matthew Hudson (D), John J. Riascos (D), Mary Schuler,

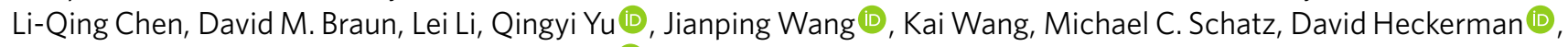
Marie-Anne Van Sluys, Glaucia Mendes Souza @, Paul H. Moore, David Sankoff, Robert VanBuren, Andrew H. Paterson, Chifumi Nagai and Ray Ming (i)

Correction to: Nature Genetics https://doi.org/10.1038/s41588-018-0237-2, published online 8 October 2018.

In the version of this article originally published, the accession codes listed in the data availability section were incorrect and the section was incomplete. The text for this section should have read "The genome assembly and gene annotation have been deposited in the NCBI database under accession number QVOL00000000, BioProject number PRJNA483885 and BioSample number SAMN09753102. The data can also be downloaded from the following link: http://www.life.illinois.edu/ming/downloads/Spontaneum_genome/.” The errors have been corrected in the HTML and PDF versions of the article. 